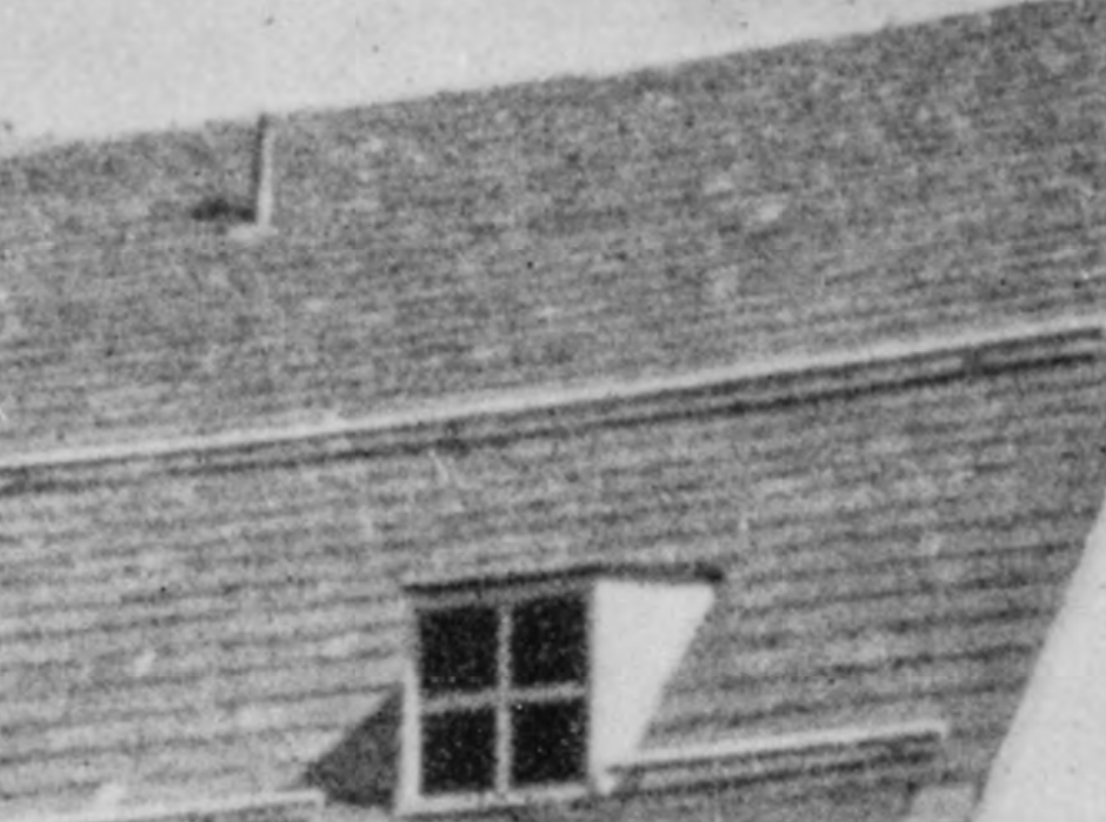

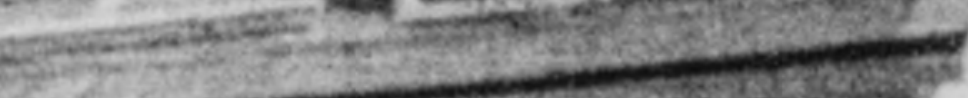

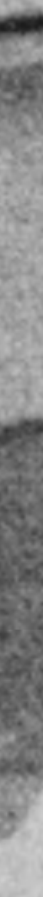

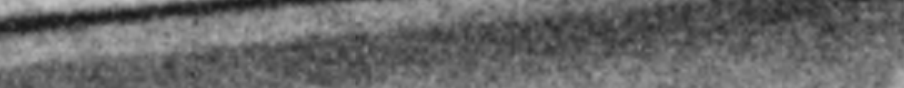

(1)

ainses

axy

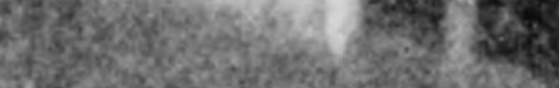

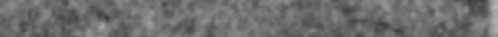

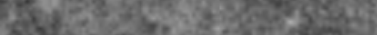

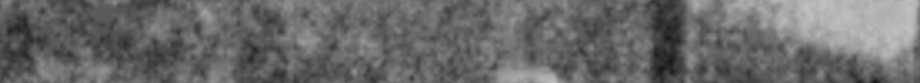
E.

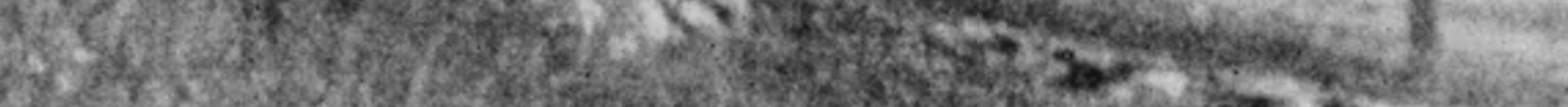

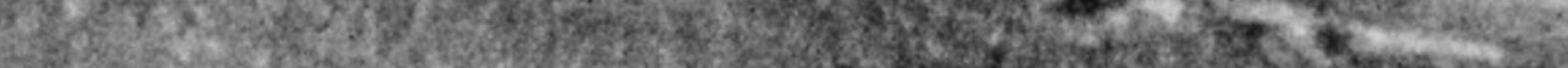




\title{
«Ma sono anche artisti». La casa Clerici di Asnago e Vender a Chiesa in Valmalenco, 1940-41
}

«But they are also artists». The Clerici house of Asnago and Vender in Chiesa Valmalenco, 1940-41

\begin{abstract}
This article investigates the critical fortune of the Clerici house, a small building built by the architects Asnago and Vender in Chiesa, in Valmalenco, between 1940 and 1941

Despite its location on the outskirts and its apparent remoteness, this type of architecture immediately found the widespread favor of the public, rightly entering the domain of emblematic modern architectures of that season, as well as of the personal poetic of the authors.

The analysis of the house's project filed for the application for planning permission seeks to investigate the critical judgements expressed by the main critics of Asnago's and Vender's work on the one hand, and to verify the possible influence of the debate on the rural and alpine house in the first half on the 20th century and of the technical and specialized public architecture between the 1930s and the 1950s on the other.

Finally, the peculiar poetic of the architects, eulogized in the project of the house, is illustrated through the comparison with other styles of architecture and with some furnishings by Asnago and Vender in the years prior to the construction of the Clerici house.
\end{abstract}

\section{Stefano Andrea Poli}

Architect, PhD in History of Architecture and Urban Planning, he teaches History of Architecture and History of Contemporary Art at the Politecnico di Milano. It carries out research, seminars and teaching activities for public and private research institutes. He has collaborated in exhibitions, conferences and volumes dedicated to Italian architecture and design in the 20th century: Piero Portaluppi (2003), Ignazio Gardella (2006), Franco Albini (2006 and 2017), Pier Luigi Nervi (2012), Mario Radice with Giuseppe Terragni, Ico Parisi and Cesare Cattaneo (2019).
Keywords

Modernity, alpine architecture, tradition, innovation, modern art. 
Il 29 aprile 1940 il podestà del comune di Chiesa in Valmalenco concede il nulla osta per la costruzione di una villa. Il progetto della pratica edilizia, consegnato poco prima, è firmato dagli architetti Mario Asnago (1896-1981) e Claudio Vender (1904-1986) per conto di Mario Clerici e di Giannina Giola Clerici, proprietaria del terreno dove la casa sarà completata l'anno seguente.

Gli scarni disegni allegati alla domanda di edificazione, piante e prospetti in scala 1:50, illustrano un edificio a pianta rettangolare, un semplice parallelepipedo sormontato da un tetto a due ripide falde simmetriche. Unica deroga alla stereometria del volume sono un'ampia loggia d'ingresso, scavata nel prospetto principale in posizione asimmetrica, e una vistosa per quanto aerea rampa di accesso che corre parallela alla stessa facciata. Articolato in un unico piano principale adibito ad abitazione, un piano interrato con rimessa e alloggio del custode e un piano sottotetto dedicato agli sport, il volume è retto da pareti perimetrali portanti e da tre pilastri portanti le travi di spina e il tetto in calcestruzzo armato.

La distribuzione interna distingue nettamente fra una zona giorno, collocata verso nord, alle spalle della loggia di ingresso, e una zona notte, allocata a sud e separata dalla prima mediante i pilastri e una serie di armadiature fisse che fungono anche da parete divisoria. La zona giorno ospita il locale principale, di cinque per cinque metri, e un andito che conduce alla scala interna, alla cucina con office e al locale per la servitù, dotato di un piccolo cesso. La zona notte, alla quale si accede direttamente dall'ambiente di soggiorno, è articolata in due camere da letto separate dal bagno. All'estrema semplicità e linearità distributiva corrisponde un'apparente omogeneità dei prospetti laterali, rispettivamente bucati da quattro finestre che illuminano le camere da letto, da tre porte-finestra aperte sulla zona giorno e da una foratura in corrispondenza della loggia. Le pareti esterne sono semplicemente intonacate e tinteggiate in bianco, mentre il tetto a capanna è rivestito in pietra a spacco, secondo la consuetudine locale, e animato da un camino e da due abbaini prismatici. più riccamente illustrate e puntualmente menzionate dalla pubblicistica.

Come si spiega la fortuna critica di una casa di montagna costruita da due architetti tutto sommato defilati rispetto all'acceso dibattito che si consumava sulle riviste di architettura?

Il breve articolo di «Domus» ne spiega in parte il successo: l'articolista utilizza la casa come esempio di modernità audace, ma che in Italia stenta ad affermarsi, al punto da sostenere che «sarebbe [...] un errore attribuire l'antico profilo [del tetto] a una qualunque nostalgia climatica. Gli architetti, anzi, nettamente reagiscono alla tentazione, o concessione che sia» (1941). Il rifiuto della citazione vernacolare, o storicista, ad eccezione della forma e del rivestimento del tetto, è utilizzato quale argomento polemico contro il principio di ambientamento. Ma Asnago e Vender disegnarono questa casa consapevoli del coevo dibattito teorico sull'architettura alpina? Si ispirarono a modelli precisi? O invece l'opera, che tuttora appare un unicum nel panorama della pubblicistica italiana dell'epoca, è più correttamente ascrivibile a una personale ricerca poetica e linguistica? Purtroppo, ignorando la costituzione della biblioteca dei due professionisti, in parte dispersa, e mancando del tutto una loro produzione scritta, è possibile avanzare solo alcune ipotesi.

Nella distribuzione interna della casa e in alcuni caratteri del linguaggio esterno si ravvisa l'eco di manuali all'epoca diffusi e probabilmente utilizzati da Asnago e Vender. Anzitutto, la netta distinzione tra zona notte e zona giorno, l'uso di armadi-parete a separare gli ambienti, la presenza di un ampio office tra cucina e soggiorno, nonostante l'angustia dei locali, e la stessa precisa organizzazione dei percorsi interni paiono trarre spunto dalle soluzioni proposte nel manuale di Enrico Agostino Griffini Costruzione Razionale della casa, edito da Hoepli in diverse versioni a partire dal 1931 (Savorra, 2000). Nel manuale Griffini trasfondeva la tassonomica conoscenza dell'architettura alpina e rustica italiana, acquisita negli anni del primo dopoguerra e raccolta in numerosi articoli, «mettendo [...] in relazione le caratteristiche fondamentali dell'abitazione moderna con la casa rustica alpina e prealpina» (Demartini, 2012). 

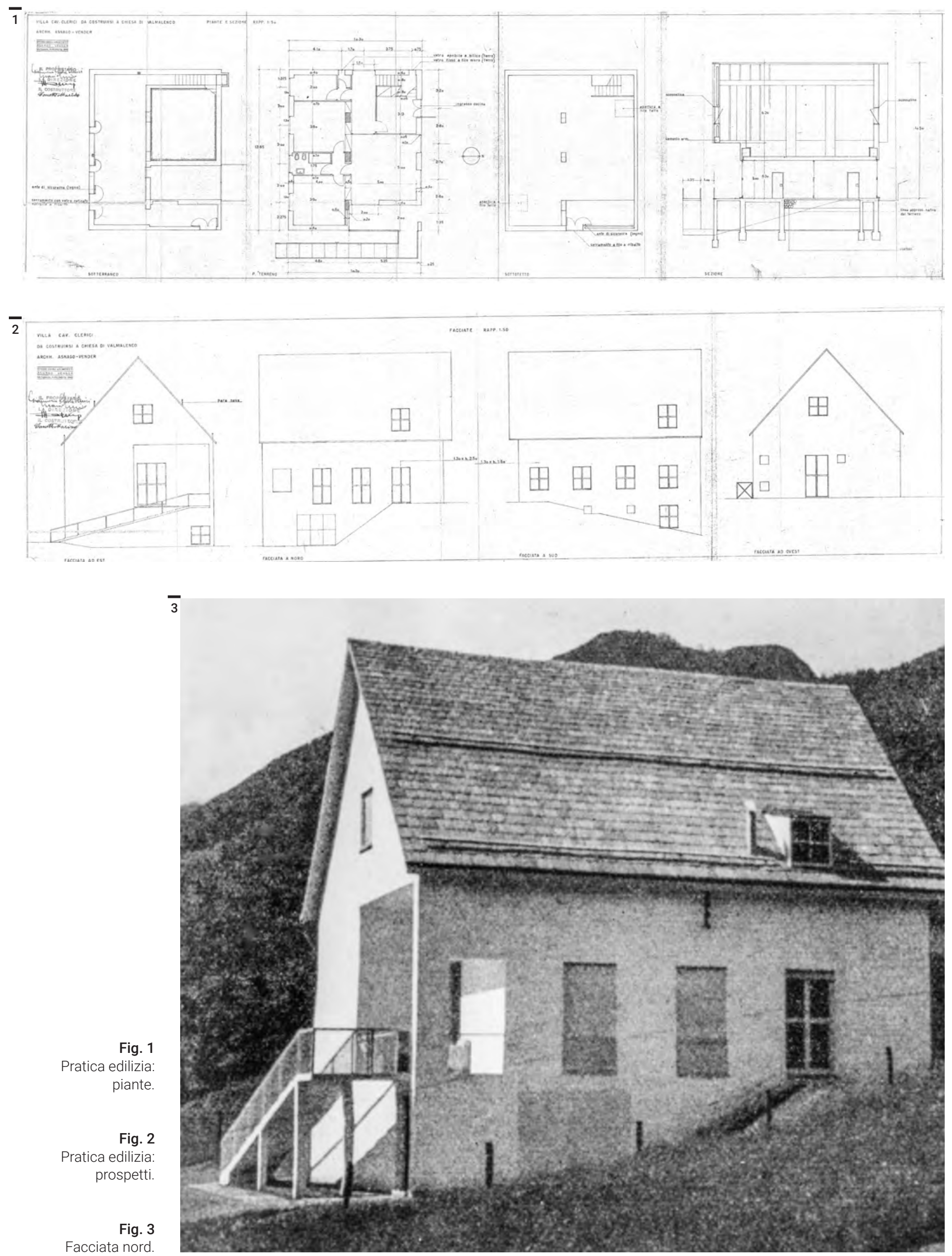


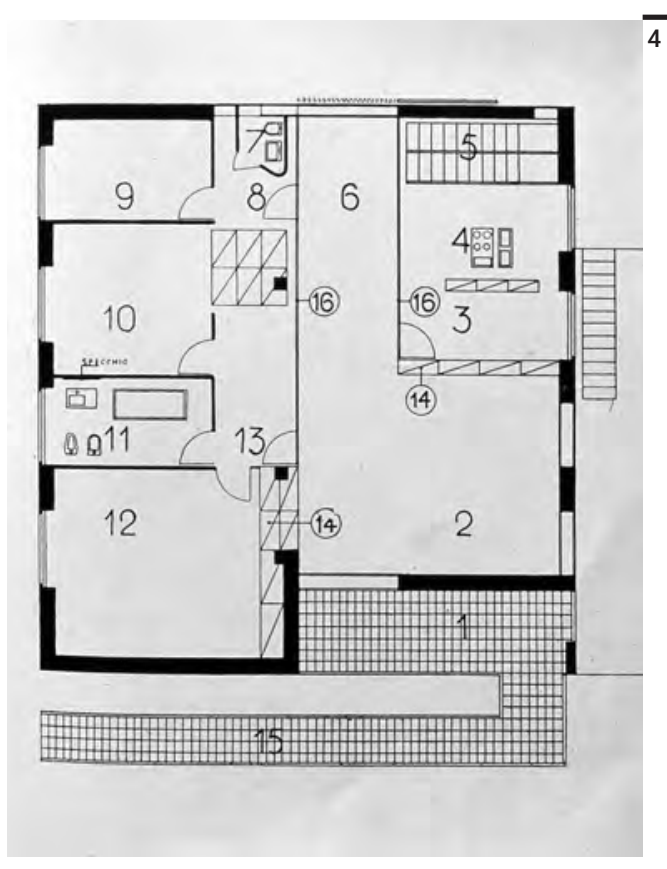

D'altro canto, l'aspetto esterno della casa è un tassello significativo per illustrare la poetica di Asnago e Vender, a partire dalla lunga pagina scritta da Gio Ponti su «Stile» (Ponti, 1943). Dai numerosi esegeti che seguirono, i due architetti e pittori sono stati giudicati capaci di conferire alle proprie architetture un carattere peculiare e inconfondibile, definito via via astratto, surreale, metafisico, purista, casto, essenziale, minimalista ante litteram, archetipico, di modanatura moderna, di ricerca tipologica, sia prendendo in prestito le tassonomie della storiografia artistica, sia coniando aggettivi e attributi nel tentativo di cristallizzare i lemmi di un repertorio ricorrente e il valore enigmatico ed impressivo della loro opera. Pare invece difficile rintracciare un rapporto con la coeva produzione di architettura alpina, sufficientemente forte da slegare la casa Clerici dalla personale ricerca degli autori. È infatti significativo che, fra le numerose illustrazioni dei volumi dedicati

Fig. 4 alle costruzioni alpine da Mario Cereghini, non Disegno di progetto: sia possibile trovare nulla di simile alla casa Cleriplanimetria. ci, né fra le architetture di civile abitazione tradini alla casa di Chiesa in Valmalenco risultano due chiese d'alta montagna, dai semplici volumi intonacati di bianco e forati da geometriche finestre Fig. 6 (Cereghini, 1950).

Vista della rampa. D'altro canto, l'osservazione dell'intero corpus di architetture di Asnago e Vender lascia emergere una predilezione per il tetto a capanna, o a padiglione,

Fig. 7

Dettaglio della rampa e della loggia di ingresso. na o rurale, mentre in città più spesso il tetto piano definisce i volumi di codomini e case in cortina.
Una relazione fra tipologia e collocazione ambientale connota in generale gli edifici dei due architetti, al di là del confronto con l'edilizia storica o popolare preesistente nel luogo di edificazione (Monestiroli, 1986).

Con casa Clerici Asnago e Vender paiono quindi seguire quel principio di semplificazione formale e geometrica che alcune avanguardie e correnti artistiche della modernità operarono in pittura come in architettura (Giolli, 1943; Airoldi, 1986). Per altri versi, la piccola abitazione di montagna non esprime un espressionismo strutturale: le forme e il disegno non illustrano alcuna teoria costruttiva, né una griglia teorica è loro impressa. Volumi e pareti praticano una reductio ad unum degli elementi funzionali e formali e sono qualificati dai vocaboli elementari del linguaggio architettonico: porte, finestre, logge, l'inattesa rampa di acceso. Asnago e Vender costruiscono la casa in cemento armato e muratura portante, con una struttura mista che sarà a lungo la più diffusa nella realtà edilizia della provincia italiana. Ma l'ordito portante e i materiali costruttivi appaiono indifferenti al risultato volumetrico, alla veste esteriore.

Forse il confronto con gli arredi di Asnago e Vender può favorire la comprensione di una attitudine progettuale che non è relegata al solo mondo dell'architettura, o della pittura. Ai mobili imprimono una forma espressiva, spinta tuttavia ben al di là del buon senso costruttivo e d'uso, il quale anzi soccombe di fronte alla necessità di purezza delle forme e di evocazione di un modello archetipico. Un quantum surreale rende gli arredi di Asnago e Vender metafore di se stessi, feticci emblematici (Giolli, 1943). Il tavolo in tondino metallico per casa Simonotti mette in forma lineare e scarna le membrature verticali e il piano orizzontale di appoggio, ma risponde alla necessità della controventatura con due tiranti a X che, secondo osservatori attenti e disinibiti quali Renato Radici e Guido Canella sono inadeguati all'uso: «Elegante ed esile questo tavolo non ha risolto felicemente l'irrigidimento della struttura; la controventatura eccessivamente bassa impedisce la libertà del movimento ai piedi dei commensali» (Canella, Radici, 1948). L'elemento di controventatura è senz'altro necessario al buon funzionamento del tavolo, ma è posizionato in un punto sbagliato, scomodo, perché la volontà estetizzante crea una forma di cartesiana pulizia, che prevale sulle necessità del buon uso.

Un processo analogo pare guidare il progetto di casa Clerici, e in parte ne spiega il sorprendente successo di critica. A colpire ancora oggi lo sguardo di un osservatore non distratto, e nutrito di cultura architettonica, è la riduzione agli elementi minimi costitutivi dell'architettura che caratteriz- 

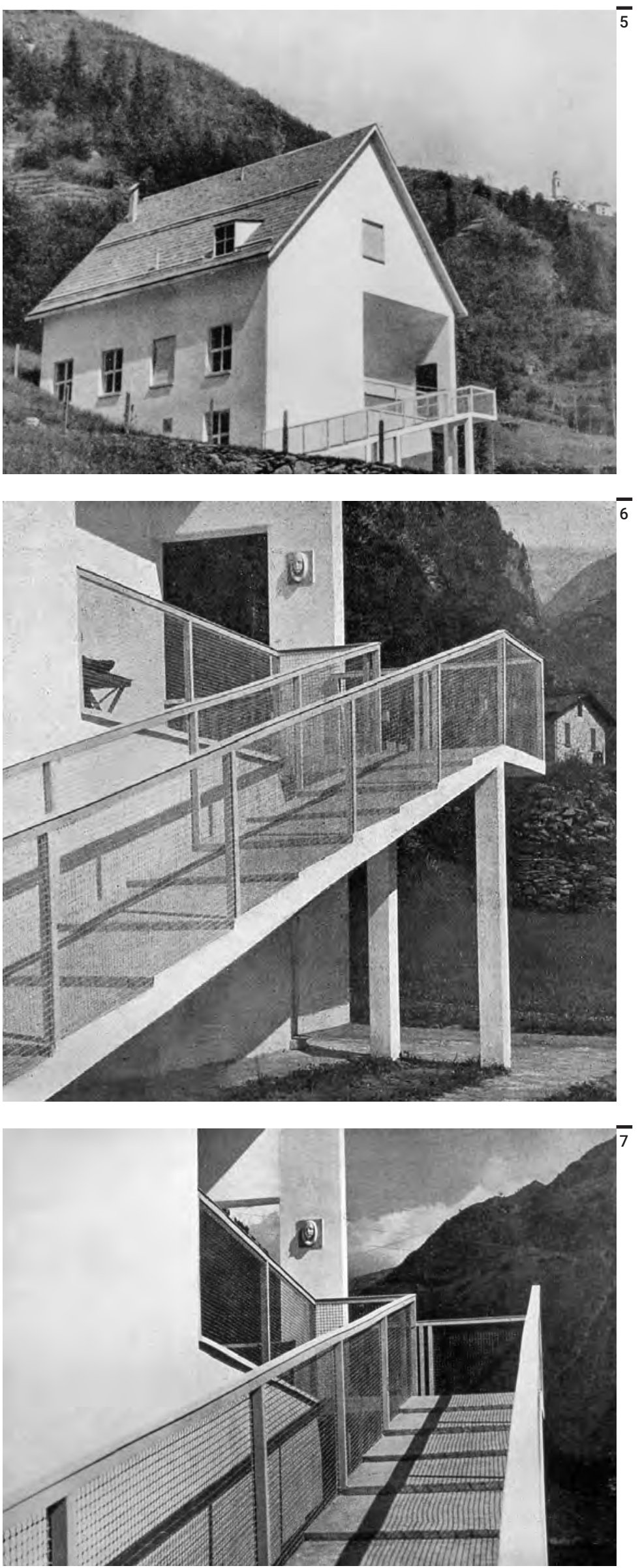

za l'insieme: tetto a falda, facciata principale a "casetta", quella atavica che un bimbo disegnerebbe, ricerca di pulizia del volume, delle superfici e dei tagli. Le forme di questa abitazione vanno oltre ogni giustificazione funzionale, ambientale, strutturale. Si giustificano da sé. Compongono un linguaggio necessario applicato alla nitidezza di tipi planimetrici e volumetrici: una sorta di ricerca della verità dell'architettura, della particella elementare, non ulteriormente divisibile, del tema assegnato al progettista (Houellebecq, 1998). Alla quale tuttavia, come nel tavolo, si applica un elemento dissonante, leit motiv manierista del linguaggio dei due architetti: la rampa, come la $\mathrm{X}$ di controventatura del mobile, è utilizzata in diversi altri edifici e, se serve a superare il dislivello tra il piano di campagna e l'ingresso, non è affatto il dispositivo più adatto, economico e confortevole per assolvere questo compito.

\section{Bibliografia}

(1941), «Casetta in montagna», in Domus, n. 168, pp. 20-23. Airoldi Renato (1986), «Asnago e Vender uno stile senza tempo», in Airoldi Renato, Mantero Enrico, Monestiroli Antonio, Albertini Antonio, Novati Massimo, Asnago/Vender Architetti, Nani, Como, 1986, pp. 13-22.

Canella Luciano, Radici Renato (1948), «Tavoli e piani d'appoggio», in Quaderni Domus, n. 6, p. 32.

Cereghini Mario (1950), Costruire in montagna, Edizioni del Milione, Milano, pp. 510-511.

Cereghini Mario (1953), Introduzione alla architettura alpina, Edizioni del Milione, Milano.

Consalez Lorenzo, Peirone Silvia (1994), Asnago e Vender. L'isolato di via Albricci a Milano, Alinea, Milano.

Demartini Elena (2012), «Gli scritti sulla casa rustica», in Camponogara Claudio, Demartini Elena, Ferrari Federico, Poli Stefano, La casa modernissima. Enrico Agostini Griffini tra sperimentazione e divulgazione, L'Ornitorinco, Milano, p. 33.

Giolli Raffaello (1943), «Architetture che fanno quadro», in Costruzioni-Casabella, n. 191-192, pp. 36-42.

Houellebecq Michel (1998), Le particules élémentaires, Flammarion, Paris.

Lissoni Alessandro (1947), Ville Casette, Gorlich, Milano, p. 84. Monestiroli Antonio (1986), «La ricerca delle forme proprie», in Airoldi Rentao, Mantero Enrico, Monestiroli Antonio, Albertini Antonio, Novati Massimo, Asnago/Vender Architetti, Nani, Como, 1986, pp. 53-60

Ponti Gio (1943), «Stile di domani. Su alcune architetture di Asnago e Vender», in Stile, n. 35, pp. 6-22.

Savorra Massimiliano (2000), Enrico Agostino Griffini, Electa, Napoli.

Zucchi Cino, Cadeo Francesca, Lattuada Monica (1999), Asnago e Vender. Architetture e progetti 1925-1970, Skira, Milano, pp. 27-28, 215.

\section{Fonti}

Archivio Asnago e Vender Milano (AAVM), Cartella AV96. 